\title{
Researching and exploiting nanomaterials useful in forensics and diagnostics
}

\section{"The contributors to this special focus issue are experts in their relative areas and represent a very diverse range of scientific disciplines, reflecting the interdisciplinary nature of the NACBO Project."}

The objective of this Nanomedicine special focus issue is to give the reader a flavor of some of the activities undertaken and results produced from the European Integrated Project, Novel and Improved Nanomaterials, Chemistries and Apparatus for Nanobiotechnology (NACBO). This project was funded by the European Commission under Framework Programme 6 and ended recently. An overview of the project, its work and achievements can be obtained from the special report by Bruce 'Novel and Improved Nanomaterials, Chemistries and Apparatus for Nanobiotechnology: the NACBO Project' in this issue [1].

Specifically, the scope of this special focus issue is to pass on to readers information with respect to the synthesis and use of some of the materials innovated, characterized and applied by the project, namely nanoparticulates made of ferrocenyl nitroxide for use as electron paramagnetic response (EPR) oximetry probes in vivo, near-infrared fluorescing nanoparticles for medical imaging, polyacrolein and poly(acrolein-costyrene) quantum dot $\mathrm{CdSe} / \mathrm{ZnS}$ composites for use in bioassays and nanoparticulate/biological materials with applications in nanomedicine and diagnostics; superparamagnetic nanoparticles/red blood cell hybrid materials as a new generation of long-lived, stable and nontoxic MRI contrast agents. It also covers new developments in forensics and microarray technology. Finally, the special focus issue attempts to pass on some of the important considerations - in principle - that NACBO investigators acquired through their project experiences (e.g., [nano]materials surface patterning and activation with organosilanes, especially aminosilanes).

\section{The contributors \& their work}

The contributors to this special focus issue are experts in their relative areas and represent a very diverse range of scientific disciplines, reflecting the interdisciplinary nature of the NACBO project. Ian Bruce, the NACBO Project Coordinator, University of Kent, Canterbury, Kent, UK has worked and published in the area of nanoparticulate silica and silica-magnetite composite materials and organosilane surface patterning for the last 18 years. In that context, for this special focus issue he has reviewed silicon, silica and amino organosilanes in terms of their biomedical relevance from a physicochemical and practical applications context [2].

Shlomo Magdassi, Head of the Casali Institute of Chemistry of the Hebrew University of Jerusalem, Israel, has been a colloid chemist for the last 30 years and in his primary research article in this issue he describes recent work by his team on the synthesis of NIR fluorescent nanoparticles that are already in pharmaceutical use and which will be beneficial in colon imaging by way of oral delivery [3].

Nick Saunders has been a senior scientist at the UK Health Protection Agency, Central Public Health Laboratory, London, UK, with a special interest in applying new technologies to microbial diagnostics, for the last 30 years. In this issue he reviews the emerging applications of nanomaterials to microarrays that are relevant to this field [4].

Mauro Magnani at the University of Urbino, Urbino, Italy, has worked on developing red blood cells for use as drug carriers for the last 20 years and reports on recent work he has been conducting on encapsulating superparamagnetic nanoparticles into red blood cells for application as MRI contrast agents [5].

Vitaly Zubov is the Head of the Polymer for Biology Laboratory at the Institute of Bioorganic Chemistry of the Russian Academy of Sciences, Moscow, Russia, and has worked and been interested in inorganic and organic nanoparticle synthesis for the last 40 years. He describes recent work by his team related to first, the

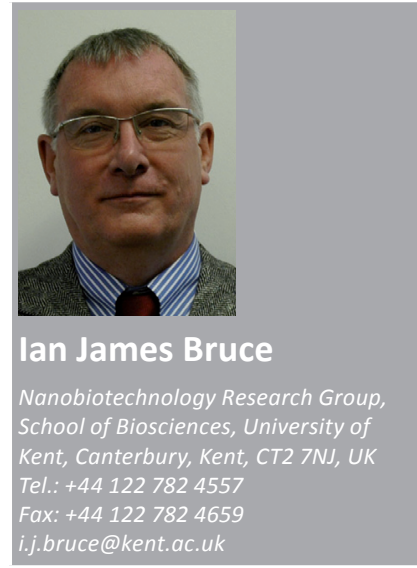

future porate $\mathrm{fSg}$ 
development of a new approach for the preparation of polymer-containing nanoparticulates useful in the one-step isolation/purification of DNA [6] and, second, the design and synthesis of uniform nanoparticulate composites formed of polymer particles of different sizes doped with semiconductor nanocrystals, which are conjugated with either full-size antibodies or recombinant mini-antibodies [7]. The work described is particularly relevant for facile sample preparation in biomedical diagnostics and research.

\section{"The benefits of the NACBO Project are already being exploited by its members and third parties with a license to exploit technologies developed by it."}

Aldo Spinella was the Director of the Italian Police Service DNA Forensics Laboratory, part of the European Network of Forensic Science Laboratories, and responsible for the development and implementation of DNA profiling by the Italian State Police Service. He and his colleagues (Emiliano Giardina and Giuseppe Novelli at Rome's Tor Vergata University) review recent developments in forensic genetics and their work in an article entitled 'From biology to the legal arena: recent advances in forensic DNA typing' [8].

Finally, Minbo Lan is Director of the Shanghai Key Laboratory of Functional Materials Chemistry and a Professor at the East China University of Science and Technology, Shanghai, China, and has interests in organic materials that can be used in EPR and its application to in vivo imaging. In his primary research article in this issue he describes recent work by his team on novel ferrocenyl nitroxide nanoparticles as EPR oximetry probes, which will be beneficial to the development of EPR oximetry in vivo [9].

\section{This special focus issue's particular benefits to readers}

The benefits of the NACBO Project are already being exploited by its members and third parties with a license to exploit technologies developed by it (see [1]). With respect to this special focus issue, its original articles give new insight into a diverse range of material syntheses and their characterization and application that should help existing and future researchers further their objectives with respect to their own work. The relevance of the NACBO work and results is also underscored by the fact that a significant part of the work of the NACBO Project is already being exploited commercially by way of joint ventures between academic and industrial partners involved in the project, new commercial start-ups, as well as by integration into existing production methodologies of industrial project partners. In summary, this special focus issue covers a very wide area in terms of subjects and interests but it is hoped that every reader will find something of interest to them.

\section{Financial \& competing interests disclosure}

This work was supported by the European Commission and the Integrated Project NACBO (contract number NMP4-CT-2004-500804). The author has no other relevant affiliations or financial involvement with any organization or entity with a financial interest in or financial conflict with the subject matter or materials discussed in the manuscript apart from those disclosed.

No writing assistance was utilized in the production of this manuscript.

\section{Bibliography}

1 Bruce IJ: Novel and Improved Nanomaterials, Chemistries and Apparatus for Nanobiotechnology: the NACBO Project. Nanomedicine (Lond.) 6(2), 187-193 (2011).

2 Rother D, Sen T, East D, Bruce IJ: Silicon, silica and its surface patterning/activation with alkoxy- and amino-silanes for nanomedical applications. Nanomedicine (Lond.) 6(2), 281-300 (2011).

3 Larush L, Magdassi S: Formation of near-infrared fluorescent nanoparticles for medical imaging. Nanomedicine (Lond.) 6(2), 233-240 (2011).
Saunders NA: Application of nanomaterials to arrays for infectious disease diagnosis. Nanomedicine (Lond.) 6(2), 271-280 (2011).

5 Antonelli A, Sfara C, Manuali E, Bruce IJ, Magnani M: Encapsulation of superparamagnetic nanoparticles into red blood cells as new carriers of MRI contrast agents. Nanomedicine (Lond.) 6(2), 211-223 (2011).

6 Kapustin DV, Prostyakova AI, Ryazantsev DY, Zubov VP: Novel composite matrices modified with nanolayers of polymers as perspective materials for separation of biomolecules and bioanalysis. Nanomedicine (Lond.) 6(2), 241-255 (2011).
7 Generalova AN, Sizova SV, Zdobnova TA et al.: Submicron polymer particles containing fluorescent semiconductor nanocrystals $\mathrm{CdSe} / \mathrm{ZnS}$ for bioassays. Nanomedicine (Lond.) 6(2), 195-209 (2011).

8 Giardina E, Spinella A, Novelli G: Past, present and future of forensic DNA typing. Nanomedicine (Lond.) 6(2), 257-270 (2011).

9 Qiu X, Zhao H, Yu R, Zhang J, Lan M: Novel ferrocenyl nitroxide nanoparticles as electron paramagnetic resonance oximetry probes in vitro and in vivo. Nanomedicine (Lond.) 6(2), 225-231 (2011). 